\title{
A Comparative Split Mouth Clinical Evaluation of Amniotic Membrane and Platelet Rich Fibrin in Patients with Gingival Recession: Two Case Reports
}

\section{Preetika Bansal*}

MDS Dasmesh Institute of Research and Dental Sciences Faridkot, Punjab, India

\begin{abstract}
Gingival recession designates the oral exposure of root surface due to the displacement of gingival margin apical to cementoenamel junction. This is split mouth design study of two patients, the first of its kind, wherein patients having bilateral isolated recession were undertaken. Both the patients were treated using Coronally Advanced Flap (CAF) with Amniotic Membrane (AM) on left side and CAF with Platelet Rich Fibrin (PRF) on right side. Recession height, width of keratinized gingiva, Probing Pocket Depth (PPD) was recorded. Patient was recalled after 3 and 6 months. The clinical outcome of these surgical procedure accounted for complete recession coverage and increase in width of keratinized gingiva, with both CAF with AM and CAF with PRF. Furthermore, the results were stable even after six months.
\end{abstract}

Keywords: Amniotic membrane; Split mouth; Coronally advanced flap; Recession; Platelet rich fibrin

\section{Introduction}

Gingival recession is an intriguing and complex phenomenon. Patients frequently are disturbed by recession. Gingival recession in anterior teeth is of huge concern due to poor esthetics and sensitivity [1].

During the last decade, great studies have been made towards predictable coverage of exposed root surfaces. Different surgical techniques have been introduced to treat gingival recession including free gingival graft, laterally positioned flap, free connective tissue graft, coronally advanced flap with connective tissue graft. In patients with a residual amount of keratinized tissue apical to the recession defect, the coronal advanced flap may be recommended [2].

Coronally advanced flap accomplishes optimum root coverage with good colour blending and complete recovery of the original soft tissue marginal morphology. This procedure does not require a donor site. Despite having low morbidity, it proves to be unstable on long term. Therefore, Coronally Advanced Flap (CAF) alone is less than optimal technique to achieve root coverage [3]. Various adjunctive agents have been used to promote healing and to further enhance the clinical outcome. Guided Tissue Regeneration (GTR) based root coverage has emerged as an alternative treatment. It not only achieves similar clinical results to those of traditional root coverage procedures, but also demonstrates histological new attachment formation [4]. The bioresorbable membranes such as collagen, human amniotic membranes, Platelet Rich Fibrin (PRF) membrane and polylactide or polyglycolide have overcome the need for second surgical procedure needed to remove non-resorbable membrane [5].

Platelet Rich Fibrin (PRF) is a fibrin 3-D polymerized matrix in which platelet, cytokines, leucocytes, growth factors and circulating stem cells are trapped and that can serve as a resorbable membrane. Thus, it can be used as a part of regeneration therapy in periodontitis [3].

Recently, amnion membrane, a third generation membrane which is a placental derived tissue containing collagen has been introduced [6]. The amnion is a membrane, building the amniotic sac that surrounds and protect an embryo. It is composed of three layers: single epithelial layer, thick basement membrane and an avascular mesenchyme. It contains no nerves, muscles or lymphatics and can be easily separated from the underlying chorion. It has been successfully used in the treatment of shallow to moderate recession defects, vestibuloplasty, periodontal intrabony defects and ridge augmentation [7].

Although, amnion membrane and PRF have been used in the treatment of gingival recession and also have shown favourable results, but split mouth study comparing both the membranes are deficient.

These two cases reported to the Dept of Periodontics, Dasmesh dental college, Faridkot. The aim of this case report is to discuss the surgical management of two patients having moderate gingival recession, with PRF and amnion membrane utilizing coronally advanced flap in split mouth design [8].

\section{Case Report}

CASE I- A 30 year old male patient complained of sensitivity. On examination, Class I recession on right upper canine (13) and left upper canine (23), the recession was $3 \mathrm{~mm}$ on both the teeth. The width of keratinized gingiva was $2 \mathrm{~mm}$ with probing pocket depth of $2 \mathrm{~mm}$ present on both the teeth (Figures 1-6).

CASE II- A 40 year old male patient revealed presence of fluorosis staining with Class III- recession present on both right upper canine (13) and left upper canine (23). The recession was $3 \mathrm{~mm}$ on 23 \& 13. Width of keratinized gingiva was $2 \mathrm{~mm}$ on both the teeth. A probing pocket depth of $4 \mathrm{~mm}$ was present on 13 and $3 \mathrm{~mm}$ on 23 (Figures 7-12).

\section{Surgical Technique}

Before the surgical technique and after the clinical investigation, scaling and root planning (Phase I therapy) was performed by ultrasonic and hand instruments and oral hygiene instructions were given to the patients. The recession present on the left side was designated as Group I and that on the right side was designated as Group II.

*Corresponding author: Dr. Preetika Bansal, Associate Professor, Department of Periodontics DIRDS, Faridkot, Punjab, India, Tel: 09814776744; E-mail: dr_preetika_11@yahoo.co.in

Received March 30, 2018; Accepted May 08, 2018; Published May 12, 2018

Citation: Bansal P (2018) A Comparative Split Mouth Clinical Evaluation of Amniotic Membrane and Platelet Rich Fibrin in Patients with Gingival Recession Two Case Reports. Dentistry 8: 487. doi:10.4172/2161-1122.1000487

Copyright: ( $\odot 2018$ Bansal P. This is an open-access article distributed under the terms of the Creative Commons Attribution License, which permits unrestricted use, distribution, and reproduction in any medium, provided the original author and source are credited. 
Citation: Bansal P (2018) A Comparative Split Mouth Clinical Evaluation of Amniotic Membrane and Platelet Rich Fibrin in Patients with Gingival Recession: Two Case Reports. Dentistry 8: 487. doi:10.4172/2161-1122.1000487

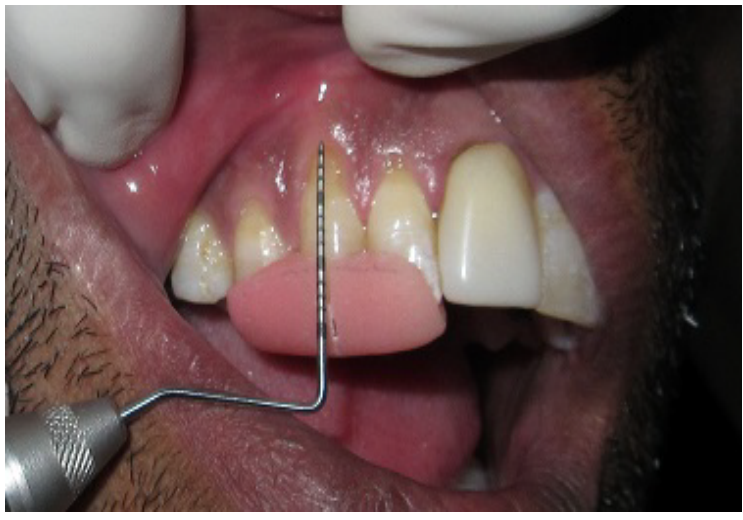

Figure 1: Pre-operative site Case1 (gp1).

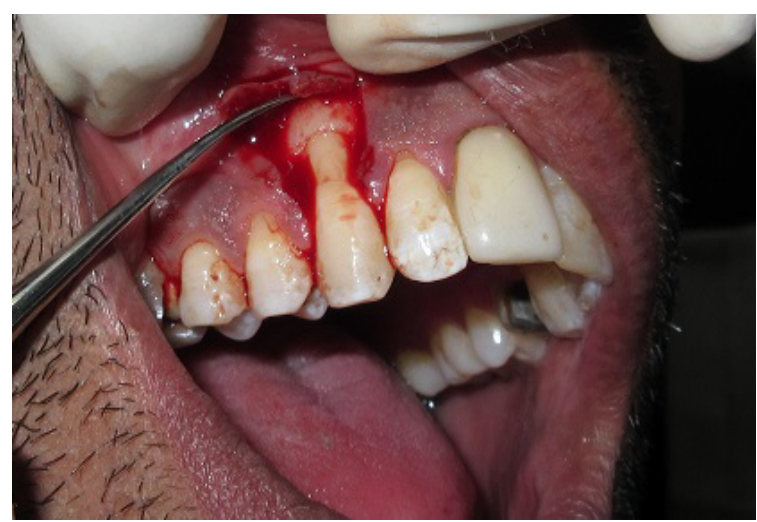

Figure 2: Flap reflection and prepared surgical site.

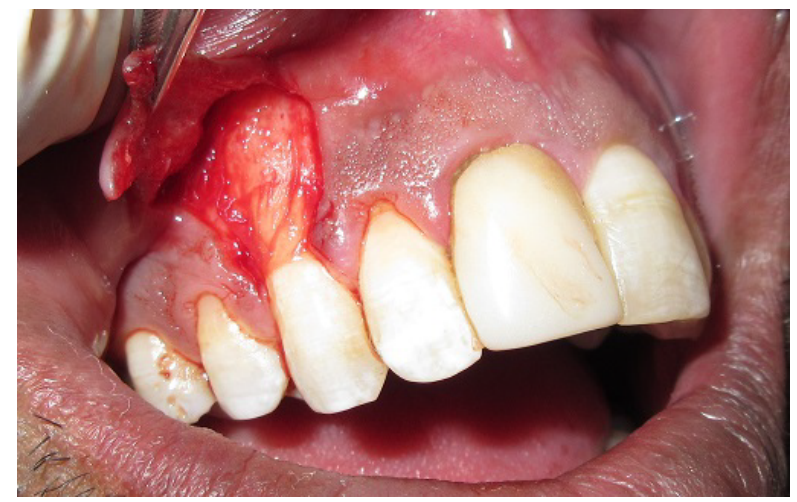

Figure 3: Amniotic membrane placed over the site.

Following administration of local anaesthesia, both groups underwent an identical coronally positioned flap technique. This was performed by raising full thickness mucoperiosteal flap using two parallel vertical incisions to outline the surgical area. An intrasulcular incision was made using a no. 15 scalpel blade to connect the two vertical incisions. The vertical incisions were made at line angles of the adjacent teeth or same teeth. The flap is positioned to the level of mucogingival junction and $1 \mathrm{~mm}$ coronal to CEJ. To facilitate coronal movement, the base of flap was undermined and separated from the periosteum with the scissors (Figures 2 and 10).

\section{In group I}

Commercially available freeze-dried, irradiated, bioabsorbable amniotic membrane of dimension 1 X $1 \mathrm{~mm}$ (Tissue Bank, Tata Memorial Hospital and Research Centre, Mumbai, India) was procured, trimmed and positioned over the root coronal to the CEJ and apically 2-3 $\mathrm{mm}$ beyond the bony margin (Figure 3 ). The flap was then coronally positioned to cover the membrane. Sling sutures were given with 4-0

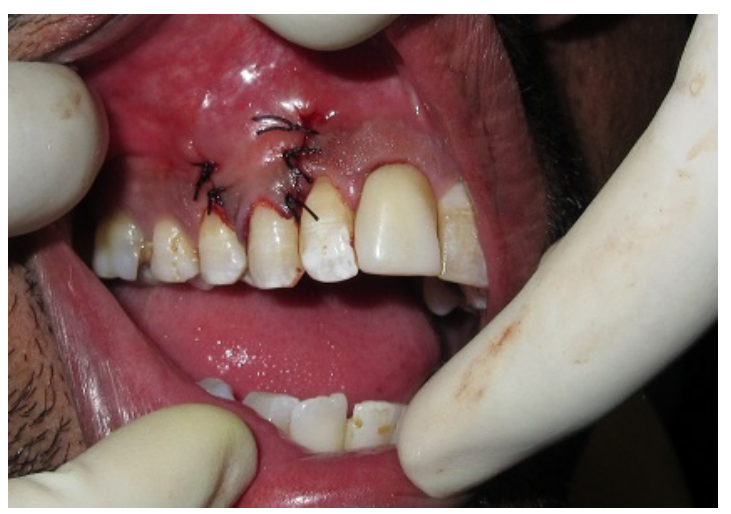

Figure 4: Flap advanced and sutured.

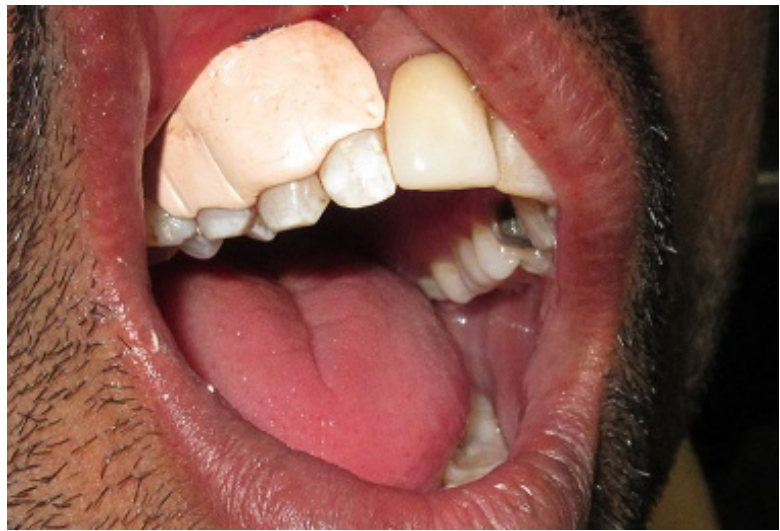

Figure 5: Coe-Pack given

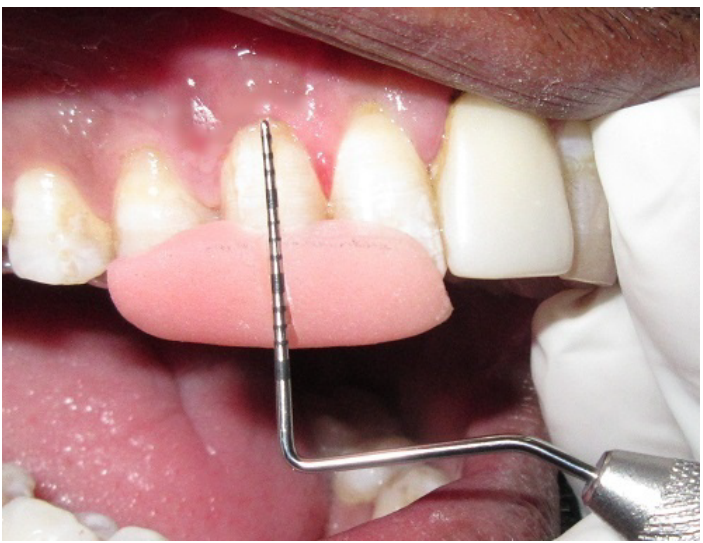

Figure 6: 6 months follow up of amniotic membrane placed Case 1 . 
Citation: Bansal P (2018) A Comparative Split Mouth Clinical Evaluation of Amniotic Membrane and Platelet Rich Fibrin in Patients with Gingival Recession: Two Case Reports. Dentistry 8: 487. doi:10.4172/2161-1122.1000487

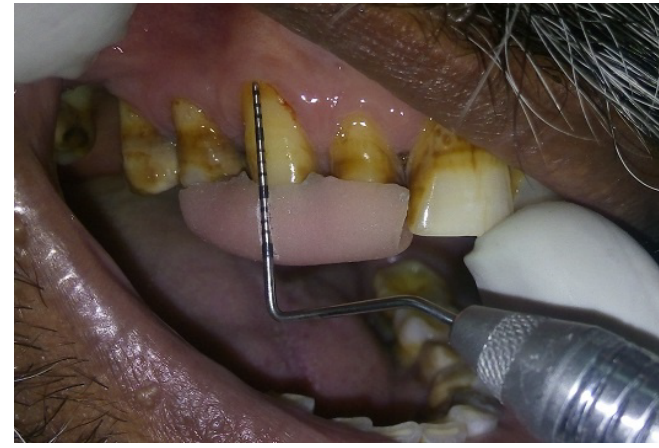

Figure 7: Pre-operative site Case 2 (gp1).

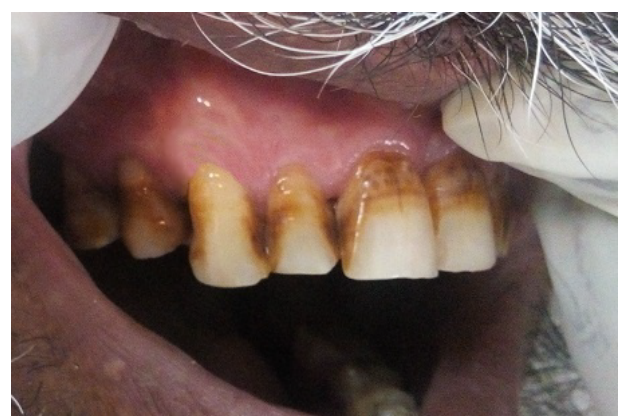

Figure 8: 6 months follow up of amniotic membrane placed Case 2.

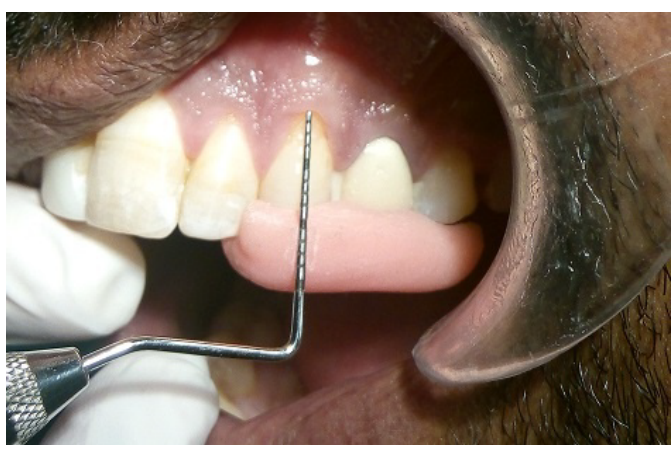

Figure 9: Pre-operative site Case 1 (gp2).

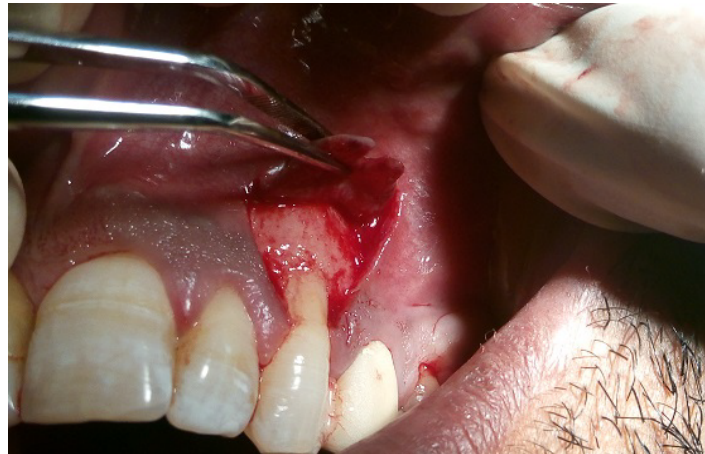

Figure 10: Flap reflection and prepared surgical site. non-resorbable braided silk suture to secure it coronally (Figure 4). The vertical releasing incisions were closed using simple interrupted suture. Gentle pressure was applied against the flap for 2-3 minutes to secure a good adaptation. A periodontal dressing (Coe-Pack) was applied to protect the surgical area for 14 days (Figure 5).

\section{In group II}

Same procedure was done but the membrane used was platelet rich fibrin (Figures 11-13). The PRF was prepared following the protocol developed by Choukron et al. Just prior to surgery, intravenous blood (by veinpuncturing of antecubital vein) was collected in $10 \mathrm{ml}$ sterile tube without anticoagulant and immediate centrifuged in the centrifugation machine at 3000 revolutions (approx. $400 \mathrm{~g}$ ) per minute

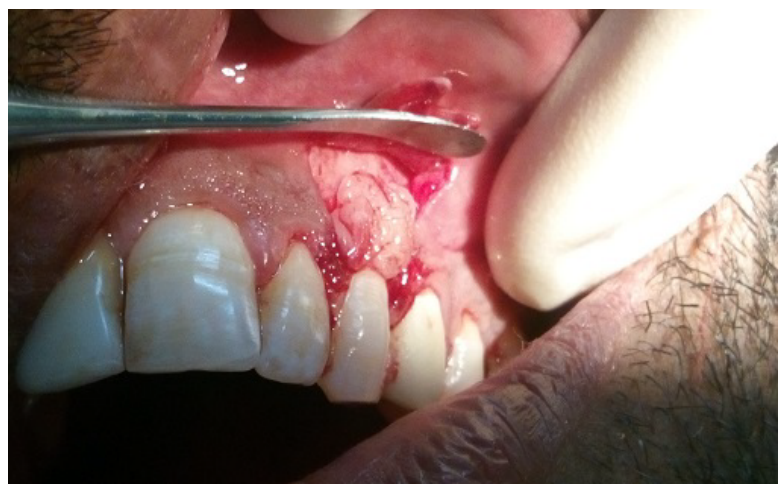

Figure 11: PRF membrane placed over the site

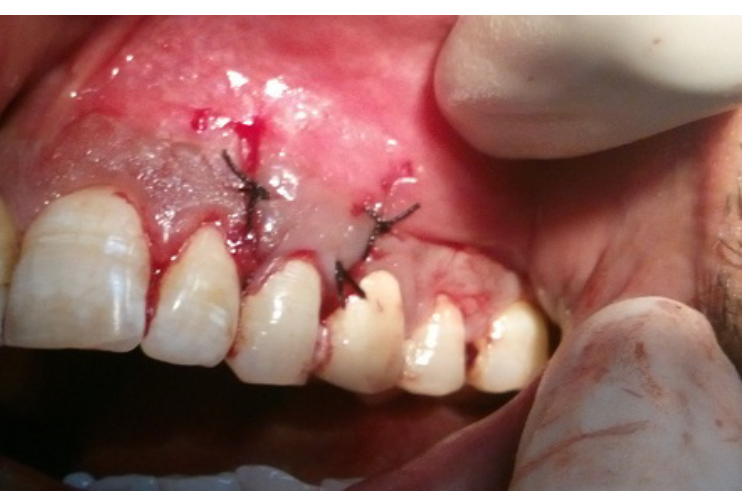

Figure 12: Flap advanced and sutured

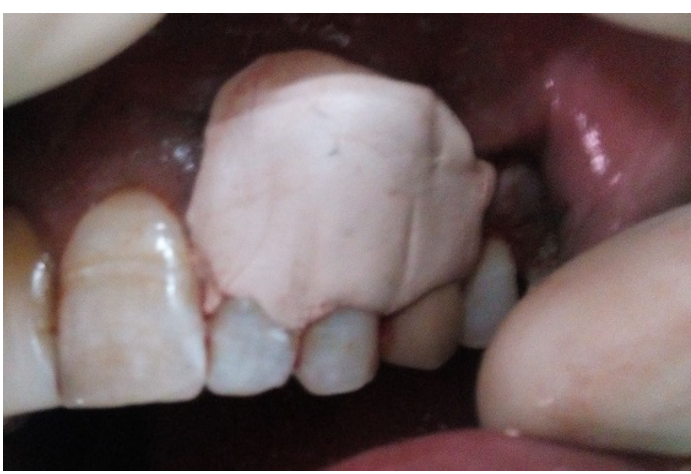

Figure 13: Coe-Pack given. 
for $10 \mathrm{~min}$. Blood centrifugation immediately after collection allows the composition of the structured fibrin clot in the middle of the tube, just between the red corpuscles at the bottom and the acellular plasma (platelet poor plasma PPP) at the top. Platelet rich fibrin was easily separated from the red corpuscles base using sterile tube tweezers and scissors just after removal of platelet poor plasma.

Patients were instructed for the care of surgical site and to report back in case of any bleeding or any adverse event. All the patients were prescribed antibiotics amoxicillin $500 \mathrm{mg} 3$ times a day for 5 days and analgesic ibuprofen $400 \mathrm{mg} 3$ times a day for 3 days. Use of $0.2 \%$ chlorohexidine digluconate mouth rinse was instructed for 4 weeks after surgery.

COE-pack and suture were removed 10 days after the surgical procedure. Patients were instructed to use soft toothbrush for mechanical plaque control. All the patients were recalled for once a week for review for the first mouth, and after 3, 6 months.

The results at recall examination after a post-operative period of 6 months revealed complete root coverage. In amniotic membrane group case I showed increase width of keratinized gingiva from $2 \mathrm{~mm}$ to 7 $\mathrm{mm}$ on 13 (Figure 6) and case II showed increase from $2 \mathrm{~mm}$ to $6 \mathrm{~mm}$ (Figure 8). Probing pocket depth of $2 \mathrm{~mm}$ was present in case I and in case II it decreased from $4 \mathrm{~mm}$ to $3 \mathrm{~mm}$.

In PRF group case I and case II both showed increase in width of keratinized gingiva from $2 \mathrm{~mm}$ to $6 \mathrm{~mm}$ on 23. A probing pocket depth in case I was $2 \mathrm{~mm}$ (Figures 14 and 15) while in case II was $3 \mathrm{~mm}$ (Figure 16).

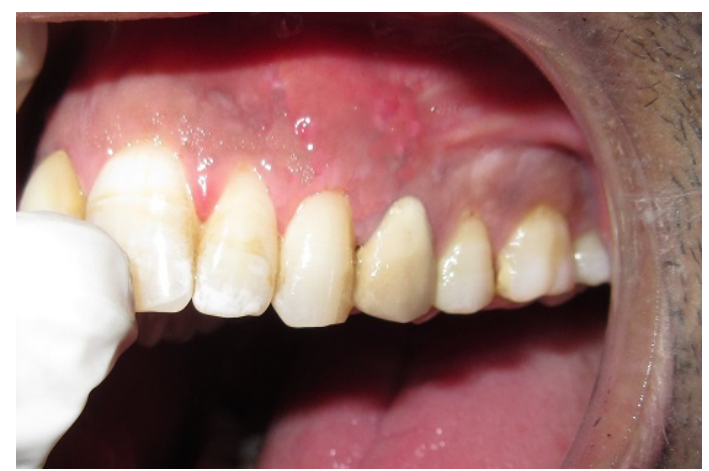

Figure 14: 6 months follow up of PRF membrane placed Case 1

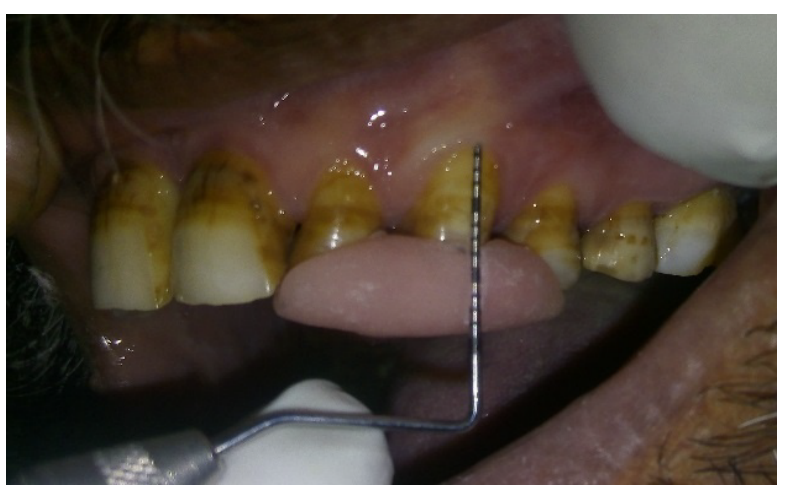

Figure 15: Pre-operative site Case 2 (gp2).

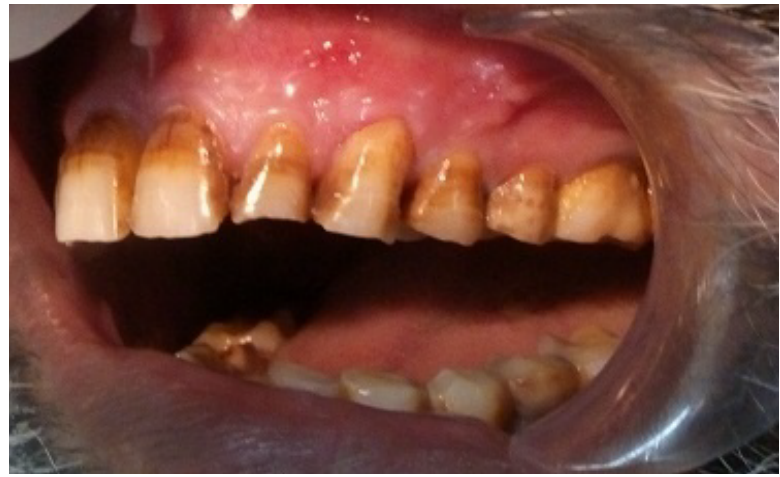

Figure 16: 6 months follow up of PRF membrane placed Case 2.

\section{Discussion}

The coronally positioned flap has long been used as a method of gaining root coverage ever since it was introduced by Norberg O (1926). It has been shown to be easy, more predictable and reliable surgical root coverage procedure. But the connective tissue attachment achieved by the coronally positioned flap is not stable over long periods. Various adjunctive agents have been used to promote healing and to further enhance the clinical outcome [9].

However, many periodontal plastic procedures used to cover denuded root surfaces have resulted in the formation of long junctional epithelium rather than regeneration, which gave birth to the principle and technique of GTR [10]. In this study, we have compared recently introduced amnion membrane- a third generation and platelet rich fibrin- a second generation membrane.

PRF is a fibrin 3-D polymerized matrix in which platelet cytokine, leucocytes, growth factors (like PDGFs, TGF $\beta$, epidermal growth factor EGF and insulin like growth factor-1, IGF-1) and presence of circulating stem cells are trapped [11,12]. It has been defined as an autologous leucocyte and platelet rich fibrin biomaterial. It was first developed by Choukroun et al. [8]. The slow polymerization process also allows the intrinsic retaining of glyconic chains and cytokines within fibrin polymers. It requires neither anticoagulant nor bovine thrombin and emerged as promising therapeutic modality in the fields of medicine and dentistry. PRF has demonstrated positive results in facial plastic surgery procedures, treatment of furcation, recession and sinus lift procedures. Thus, a physiological architecture that is very favourable to the healing process is obtained with platelet rich fibrin.

Amnion membrane is a composite membrane consisting of a pleuripotent cellular element embedded in a semi permeable membranous structure, which is a placental derived tissue containing collagen. Human amnion lines the innermost portion of the amniotic sac is $100-200 \mu$ in thickness. It is an immune tolerant structure. Also, the existence of pleuripotent stem cells possessing the ability of transdifferentiation to other cellular elements of periodontium makes it suitable for GTR. Excellent revascularization and provision of rich source of stem cells demonstrate that Amnion membrane enhances gingival wound healing. The basement membrane contains collagen type III, IV and V and cell adhesion bioactive factors including fibronectin and laminins (mainly Laminin 3). It helps in soft tissue healing, modulates angiogenesis, cicatrisation, epithelization and facilitated migration [13].

Amnion membrane has been used in the field of oral surgery from 
Citation: Bansal P (2018) A Comparative Split Mouth Clinical Evaluation of Amniotic Membrane and Platelet Rich Fibrin in Patients with Gingival Recession: Two Case Reports. Dentistry 8: 487. doi:10.4172/2161-1122.1000487

1969 onwards. Its applications in post traumatic orbital surgery and temporomandibular joint surgery have been added since 2010. Tissue engineering with amnion membrane is a fast expanding field with a high variety of future options [14]. This membrane is available as wound dressing material for surgical wounds of tongue and buccal mucosa. Amnion has been successfully used in the treatment of the shallow to moderate recession defects, vestibuloplasty and ridge augmentation.

In this study, a split mouth design was undertaken. All the cases of Group I and Group II showed uncomplicated and rapid healing. Significant improvements were observed in gingival recession reduction, probing pocket depth and width of attached gingival in both groups from baseline to 3 months to 6 months. In the amnion group the results are found to be similar with the studies done by Ghahroudi et al. [15] and Gurinsky [7] who concluded that amnion membrane have comparable results as connective tissue graft and can be used as an alternative to autogenous grafts respectively. Shah et al. [13] in a case report observed enhanced wound healing and esthetics with amnioin membrane.

In PRF group results were in accordance with Reddy et al. [16] who reported enhanced root coverage with increase in thickness of gingiva. Padma et al. [17] found superior root coverage with use of PRF along with CAF.

These results might be due to the property of Platelet rich fibrin to progressively release cytokines during fibrin matrix remodeling in the process of healing [17] and that of Amnion membrane can be attributed to induction of fibroblast proliferation and presence of vascular growth factor $[18,19]$. The increase in thickness of gingiva in Amnion membrane is due to the presence of keratinocyte growth factor which might promote keratinization of epithelial cells and help in mucogingival junction in maintaining its position $[15,20]$.

\section{Conclusion}

It can be concluded that both, recently introduced amnion membrane ( $3^{\text {rd }}$ generation membrane ) and platelet rich fibrin membrane $\left(2^{\text {nd }}\right.$ generation membrane) are equally efficacious in the treatment of gingival recession and use of these resorbable membranes with coronally advanced flap is a versatile treatment modality for root coverage of isolated gingival recessions. However, amnion membrane exhibited certain additive advantages over the PRF membrane such as better handling properties as it is comparatively thin, enabling it to mould according to the defect anatomy and root surfaces easily and alternate to PRF in reducing the need for preparation of autologous biomaterial.

\section{References}

1. Kassab MM, Badawi H, Dentino AR (2010) Treatment of gingival recession Dental clinics of North America 54: 129-140.

2. Lee EJ, Meraw SJ, Oh TJ, Giannobile WV, Wang HL (2002) Comparative histologic analysis of coronally advanced flap with and without collagen membrane for root coverage. Journal of periodontology 73: 779-788.
3. Tanya J, Thomas BS (2012) Platelet rich fibrin membrane for recession coverage. J Dent 2: 223-237.

4. Malathi KG, Dev JN, Kumar KS, Srikanth C, Chandra PR, et al. (2013) A clinical evaluation of a bioresorbable membrane and porous hydroxyapatite in the treatment of human molar class II furcations. Journal of Indian Society of Periodontology 17: 617.

5. Elgali I, Omar O, Dahlin C, Thomsen P (2017) Guided bone regeneration: materials and biological mechanisms revisited. Eur J Oral Sci 125: 315-337.

6. Holtzclaw DJ, Toscano NJ (2013) Amnion-chorion allograft barrier used for guided tissue regeneration treatment of periodontal intrabony defects: A retrospective observational report. Clinical Advances in Periodontics 3 : 131-137.

7. Gurinsky B (2009) A novel dehydrated amnion allograft for use in the treatment of gingival recession: An observational case series. The Journal of Implant \& Advanced Clinical Dentistry 1: 65-73.

8. Choukroun J, Adda F, Schoeffler C, Vervelle A (2001) An opportunity in perimplantology: the PRF. Implantodontic 42: 55-56.

9. Avinash K, Selvan T (2014) Coronally advanced flap in the treatment of recession coverage. Int J Dent Case Rep 4: 1-10.

10. Lee EJ, Meraw SJ, Oh TJ, Giannobile WV, Wang HL (2002) Comparative histologic analysis of coronally advanced flap with and without collagen membrane for root coverage. J Periodontol 73: 779-788.

11. Bajaj P, Agarwal E, Rao NS, Naik SB, Pradeep AR, et al. (2017) Autologous Platelet-Rich Fibrin in the treatment of 3-Wall Intrabony defects in aggressive periodontitis: A Randomized Controlled Clinical Trial. J Periodontol 88: 1186-1191.

12. Choukroun J, Diss A, Simonpieri A, Girard MO, Schoeffler C, et al. (2006) Platelet-rich fibrin (PRF): a second-generation platelet concentrate. Part IV: clinical effects on tissue healing. Oral surgery, oral medicine, oral pathology, oral radiology and endodontics 101: 56-60.

13. Shah R, Sowmya NK, Mehta DS (2014) Amnion membrane for coverage of gingival recession: A novel application. Contemporary clinical dentistry 5: 293.

14. Kesting MR, Wolff KD, Nobis CP, Rohleder NH (2014) Amniotic membrane in oral and maxillofacial surgery. Oral Maxillofac Surg 18: 153-164.

15. Ghahroudi AA, Khorsand A, Rokn AR, Sabounchi SS, Shayesteh YS, et al (2013) Comparison of amnion allograft with connective tissue graft for root coverage procedures: a double-blind, randomized, controlled clinical trial. Journal of the International Academy of Periodontology 15: 101-112.

16. Reddy SP, Prasad MGS, Agnihotri J, Anuradha D, Singh S, et al. (2013) Management of multiple recession defect using modified coronally advanced flap alone or with PRF. Int J Health Sci Res 3: 133-138.

17. Padma R, Shilpa A, Kumar PA, Nagasri M, Kumar C, et al. (2013) A split mouth randomized controlled study to evaluate the adjunctive effect of platelet-rich fibrin to coronally advanced flap in Miller's class-I and II recession defects. J Indian Soc Periodontol 17: 631-636.

18. Betancourt P, Elgueta R, Fuentes R (2017) Treatment of endo-periodontal lesion using leukocyte-platelet-rich fibrin. A case report. Colomb Med 48 204-207.

19. Mahajan R, Gill AS, Khinda PK, Shewale A, Saravanan SP (2015) Guided Tissue Regeneration Based Treatment of Root Coverage using Placental Membrane Allograft: A Case Report. IJSS Case reports and reviews 2: 1-5.

20. Pundir AJ, Agrawal V, Pundir S, Diwan V, Bodh S (2016) Comparative Evaluation of the Efficacy of Human Chorion and Amnion with Coronally Advanced Flap for Recession Coverage: A Case Series. Clin Adv Periodontics 6: 118-126. 